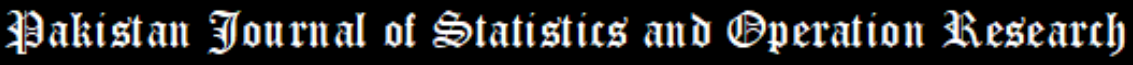

\section{Estimation \\ Methods of Alpha Power Exponential Distribution with Applications to Engineering and Medical Data}

\author{
Mazen Nassar ${ }^{1,2}$, Ahmed Z. Afify ${ }^{3 *}$, Mohammed K. Shakhatreh ${ }^{4}$ \\ ${ }^{*}$ Corresponding Author
}

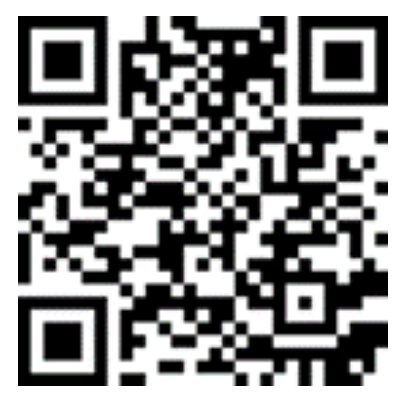

1. Department of Statistics, Faculty of Science, King Abdulaziz University, Jeddah, Saudia Arabia

2. Department of Statistics, Faculty of Commerce, Zagazig University, Egypt mezo10011@ gmail.com

3. Department of Statistics, Mathematics and Insurance, Benha University, Egypt ahmed.afify@ fcom.bu.edu.eg

4. Department of Mathematics and Statistics Jordan University of Science and Technology, Jordan mkshakhatreh6@ just.edu.jo

\begin{abstract}
This paper addresses the estimation of the unknown parameters of the alpha power exponential distribution (Mahdavi and Kundu, 2017) using nine frequentist estimation methods. We discuss the finite sample properties of the parameter estimates of the alpha power exponential distribution via Monte Carlo simulations. The potentiality of the distribution is analyzed by means of two real data sets from the fields of engineering and medicine. Finally, we use the maximum likelihood method to derive the estimates of the distribution parameters under competing risks data and analyze one real data set.
\end{abstract}

Key Words: Alpha power transformation; hazard rate function; maximum likelihood estimation; method of maximum product spacing; simulation.

Mathematical Subject Classification: 60E05, 62E15.

\section{Introduction}

Several families of distributions have been constructed by extending common well-known distributions by adding one or more parameters to the baseline distribution. These generalized classes of distributions provide more flexibility in modeling data in applied areas such as economics, engineering, lifetime analysis, insurance, finance and environmental sciences.

Mahdavi and Kundu (2017) introduced a new method for generating distributions by adding an extra shape parameter to a well known baseline distributions. The new method is called alpha power transformation (APT) method. Let $G(x)$ be the cumulative distribution function (CDF) of a continuous random variable $X$, then the APT of $G(x)$ is defined as

$$
F(x)=\left\{\begin{array}{lll}
\frac{\alpha^{G(x)}-1}{\alpha-1} & \text { if } \quad \alpha>0, \alpha \neq 1 \\
G(x) & \text { if } \quad \alpha=1,
\end{array}\right.
$$

and the corresponding probability density function (PDF) is

$$
f(x)=\left\{\begin{array}{lll}
\frac{\log \alpha}{\alpha-1} g(x) \alpha^{G(x)} & \text { if } \quad \alpha>0, \alpha \neq 1 \\
G(x) & \text { if } \quad \alpha=1
\end{array}\right.
$$


Many authors considered the APT method to extend some univariate distributions as particular cases. Mahdavi and Kundu (2017) introduced the alpha power exponential (APE) distribution by taking the exponential baseline distribution and studied the main properties as well as the parameters estimation of the proposed distribution. Nassar et al. (2017) introduced the alpha power Weibull distribution and showed that the new distribution gives better modelling than some other generalizations of the Weibull distribution using two real data sets. Dey et al. (2017) used the generalized exponential baseline distribution and the APT method to introduce alpha power generalized exponential (APGE) distribution. Nadarajah and Okorie (2018) derived a closed form expressions for moment properties of the APGE distribution. Nassar et al. (2018b) introduced a new family of distributions based on the APT method by inverting the quantile function of any distribution as a function of the APT CDF given by (1). Dey et al. (2018a, 2018b) studied the cases when $G(x)$ correspond to inverse Lindley and Lindley distributions, respectively. Mead et al. (2019) studied the general mathematical properties of the APT family and proposed the alpha power exponentiated Weibull distribution. Nassar et al. (2019) proposed a new generalization of the APT class called Marshall Olkin alpha power family.

It is well-know that the maximum likelihood estimation and moment estimation methods are classical methods of estimation. There are other estimation methods have been proposed in the literature, as alternatives to the classical estimation methods, namely, the L-moments estimator, ordinary least squares estimator, weighted least squares estimator, percentile estimator, maximum product of spacing estimator and Minimum distance methods including Cramér-von Mises estimator and Anderson-Darling estimator. However, these methods do not have good theoretical properties, they can provide better estimates, in some cases, than the maximum likelihood estimation and moment estimation methods.

Our aim in this article is to consider nine different frequentist estimators for the two-parameter APE distribution and evaluates their performance for different sample sizes and different parameter values. Furthermore, we provide three applications to real data, one of them is applied to competing risks data. Many authors have compared several classical estimation methods for estimating the parameters of well-known distributions. For example, Nassar et al. (2018c) for transmuted exponentiated Pareto, Sen et al. (2019) for the quasi xgamma-geometric, Shakhatreh et al. (2019) for the generalized extended exponential-Weibull, Afify et al. (2020) for the Weibull Marshall-Olkin Lindley, and Afify and Mohamed (2020) for the extended odd Weibull exponential distributions.

The rest of the paper is organized as follows. In Section 2, we discuss the APE distribution. Nine estimation methods are considered in Section 3. The performance and comparison of these methods in terms of mean relative estimates and root mean-squared error are provided in Section 4. The practical importance of the APE model is illustrated by analyzing two real data sets in Section 5. In Section 6, the APE distribution is applied to competing risks analysis and one real data set is used to show the flexibility of the APE distribution in modelling competing risks data. Finally, some concluding remarks are given in Section 7.

\section{Alpha Power Exponential Distribution}

Let $X$ follows the exponential random variable with scale parameter $\lambda>0$, then the CDF of the APE distribution is given by

$$
F(x)=\left\{\begin{array}{lll}
\frac{\alpha^{\left(1-e^{-\lambda x}\right)}-1}{\alpha-1} & \text { if } & \alpha \neq 1 \\
1-e^{-\lambda x} & \text { if } & \alpha=1
\end{array}\right.
$$

and the corresponding PDF is

$$
f(x)=\left\{\begin{array}{lll}
\frac{\lambda \log \alpha}{\alpha-1} e^{-\lambda x} \alpha^{\left(1-e^{-\lambda x}\right)} & \text { if } & \alpha \neq 1 \\
\lambda e^{-\lambda x} & \text { if } & \alpha=1
\end{array}\right.
$$

The survival and the hazard rate functions for the APE distribution, respectively, are in the following forms

$$
\bar{F}(x)= \begin{cases}\frac{\alpha}{\alpha-1}\left(1-\alpha^{-e^{-\lambda x}}\right) & \text { if } \quad \alpha \neq 1 \\ e^{-\lambda x} & \text { if } \quad \alpha=1\end{cases}
$$

and

$$
h(x)=\left\{\begin{array}{lll}
\frac{\lambda \log (\alpha) e^{-\lambda x} \alpha^{-e^{-\lambda x}}}{1-\alpha^{-e^{-\lambda x}}} & \text { if } \quad \alpha \neq 1 \\
\lambda & \text { if } \quad \alpha=1
\end{array}\right.
$$


The quantile function of the APE distribution is given by

$$
Q(p)=\frac{-1}{\lambda} \log \left\{\frac{\log \left[\frac{\alpha}{1+p(\alpha-1)}\right]}{\log \alpha}\right\},
$$

where $0<p<1$. Also, the $r$ th moments about zero can be expressed as follows

$$
E\left(X^{r}\right)=\frac{\alpha r !}{\lambda^{r}(1-\alpha)} \sum_{k=1}^{\infty} \frac{(-\log \alpha)^{k}}{k^{r} k !} .
$$

Mahdavi and Kundu (2017) showed that the $f(x)$ in (4) is a decreasing function of $x>0$ if $\alpha \leq e$, and for $\alpha>e$, $f(x)$ is a unimodal function with mode at $\log (\log \alpha) / \lambda$. They also proved that the hazard rate function in (6) is a decreasing function of $x>0$ when $\alpha<1$, and for $\alpha>1$ it is an increasing function of $x>0$. Figure 1 displays the various curves for the PDF and hazard rate functions of a APE distribution for $\lambda=1$ and various values of $\alpha$.

Mahdavi and Kundu (2017) studied the various properties of the APE distribution including explicit expressions of moments, moment generating function, order statistics and expression of the entropies. They used the method of maximum likelihood to estimate the unknown parameters and analyzed one real data set to show how the APE distribution works in practice.

(a)

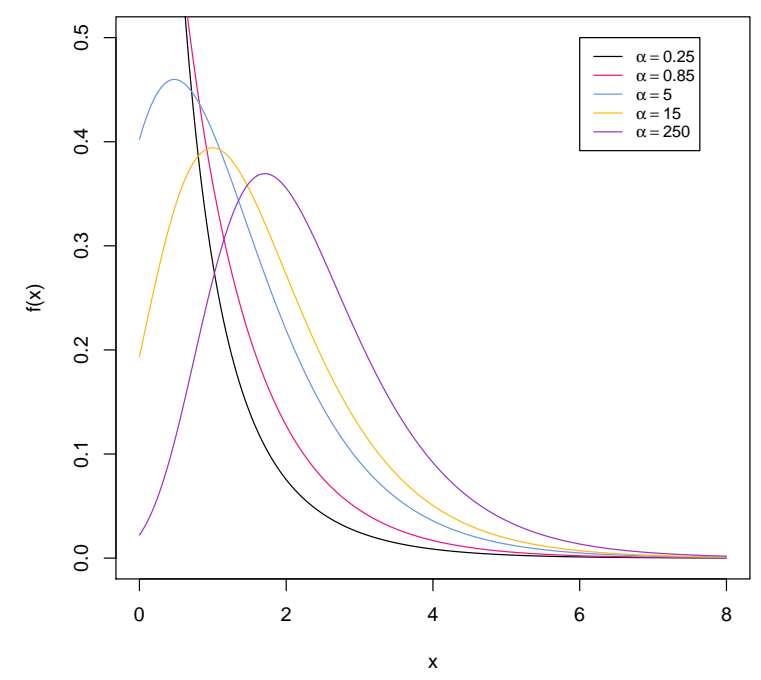

(b)

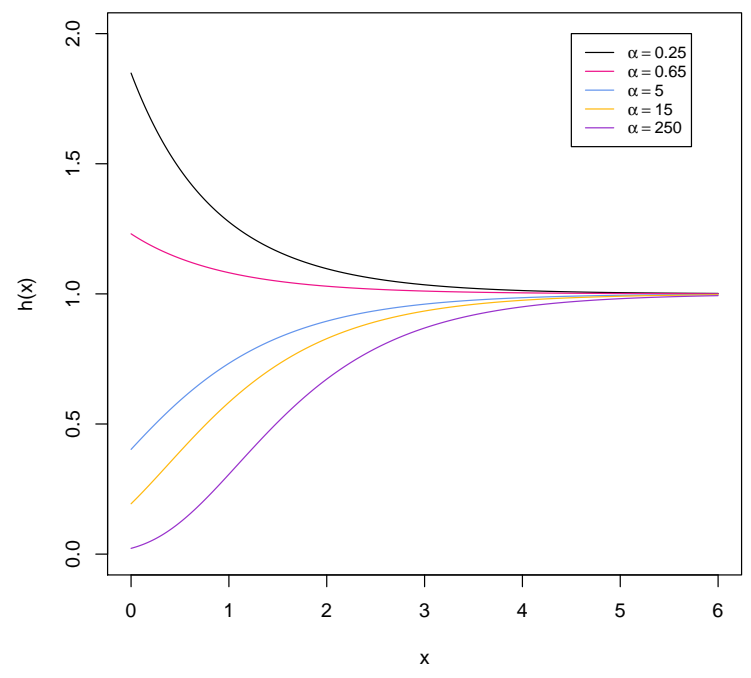

Figure 1: (a) The pdf and (b) the hazard rate function of the APE distribution for $\lambda=1$ and different values of $\alpha$.

\section{Methods of Estimation}

In this section, we consider nine methods of estimation to estimate the unknown parameters of the APE distribution. The used estimation methods are (1) maximum likelihood method, (2) methods of moments, (3) methods of L-moments, (4) ordinary least squares, (5) weighted least squares, (6) method of percentile, (7) maximum product of spacing method, (8) method of Cramér-von-Mises and (9) method of Anderson-Darling. 


\subsection{Maximum likelihood}

Let $x_{1}, x_{2}, \cdots, x_{n}$ be a random sample of size $n$ from APE distribution, then from (4) we can write the log-likelihood function as follows

$$
L(\alpha, \lambda)=n \log \alpha+n \log \lambda+n \log \left(\frac{\log \alpha}{\alpha-1}\right)-\lambda \sum_{i=1}^{n} x_{i}-(\log \alpha) \sum_{i=1}^{n} e^{-\lambda x_{i}},
$$

Mahdavi and Kundu (2017) derived the two normal equations and obtained the the maximum likelihood estimate (MLE) of $\alpha$ denoted be $\hat{\alpha}_{M L E}$ for fixed $\lambda$ as

$$
\hat{\alpha}_{M L E}(\lambda)=\exp \left\{\frac{\sum_{i=1}^{n} x_{i}-\frac{n}{\lambda}}{\sum_{i=1}^{n} x_{i} e^{-\lambda x_{i}}}\right\},
$$

and then obtained the MLE of $\lambda$ denoted be $\hat{\lambda}_{M L E}$ by solving

$$
\hat{\lambda}_{M L E}=1+\frac{\left(\hat{\alpha}_{M L E}(\lambda)-1-\hat{\alpha}_{M L E}(\lambda) \log \hat{\alpha}_{M L E}(\lambda)\right)}{\hat{\alpha}_{M L E}(\lambda)\left(\hat{\alpha}_{M L E}(\lambda)-1\right) \log \hat{\alpha}_{M L E}(\lambda)}-\frac{1}{n} \sum_{i=1}^{n} e^{-\lambda x_{i}},
$$

upon obtaining $\hat{\lambda}_{M L E}$ from (11), then $\hat{\alpha}_{M L E}$ can be obtained from (10) as $\hat{\alpha}_{M L E}=\hat{\alpha}_{M L E}\left(\hat{\lambda}_{M L E}\right)$.

\subsection{Methods of moments}

The moment estimates (MEs) of $\alpha$ and $\lambda$, denoted by $\hat{\alpha}_{M E}$ and $\hat{\lambda}_{M E}$, of the APE distribution can be obtained by equating the first two sample moments with the first two theoretical moments obtained from (8). The $\hat{\alpha}_{M E}$ and $\hat{\lambda}_{M E}$ can be obtained by solving

$$
\frac{1}{n} \sum_{i=1}^{n} x_{i}-\frac{\alpha}{\lambda(1-\alpha)} \sum_{k=1}^{\infty} \frac{(-\log \alpha)^{k}}{k k !}=0
$$

and

$$
\frac{1}{n} \sum_{i=1}^{n} x_{i}^{2}-\frac{2 \alpha}{\lambda^{2}(1-\alpha)} \sum_{k=1}^{\infty} \frac{(-\log \alpha)^{k}}{k^{2} k !}=0 .
$$

From (12) and for fixed $\alpha$ we can obtain $\hat{\lambda}_{M E}(\alpha)$ as

$$
\hat{\lambda}_{M E}(\alpha)=\frac{n\left\{\frac{\alpha}{(1-\alpha)} \sum_{k=1}^{\infty} \frac{(-\log \alpha)^{k}}{k k !}\right\}}{\sum_{i=1}^{n} x_{i}},
$$

then $\hat{\alpha}_{M E}$ can be obtained by solving the following nonlinear equation

$$
\frac{1}{n} \sum_{i=1}^{n} x_{i}^{2}-\frac{2 \alpha}{\hat{\lambda}_{M E}(\alpha)^{2}(1-\alpha)} \sum_{k=1}^{\infty} \frac{(-\log \alpha)^{k}}{k^{2} k !}=0 .
$$

Once $\hat{\alpha}_{M E}$ is obtained from (15), $\hat{\lambda}_{M E}$ can be obtained directly from (14).

\subsection{Methods of L-moments}

The method of L-moments (LM) was introduced by Hosking (1990) to estimate the unknown parameters on the basis of linear combinations of order statistics. Let $x_{1: n}<x_{2: n}<\cdots<x_{n: n}$ be the order statistics of a sample of size $n$ from the APE distribution, then from Hosking (1990), the first two sample L-moments, respectively, are

$$
\ell_{1}=\bar{x} \quad \text { and } \quad \ell_{2}=\frac{2}{n(n-1)} \sum_{i=1}^{n}(i-1) x_{i: n}-\bar{x}
$$


The first two population L-moment for the APE distribution can be obtained as follow

$$
\tau_{1}=E\left(x_{1: 1}\right)=\int_{0}^{1} Q(u \mid \alpha, \lambda) d u \text { and } \tau_{2}=\frac{1}{2}\left[E\left(x_{2: 2}\right)-E\left(x_{1: 2}\right)\right]=\int_{0}^{1} Q(u \mid \alpha, \lambda)(2 u-1) d u .
$$

where $Q(u \mid \alpha, \lambda)$ is the quantile function of the APE distribution given by (7). The L-moment estimates of $\alpha$ and $\lambda$, denoted by $\hat{\alpha}_{L M}$ and $\hat{\lambda}_{L M}$ can be obtained by equating the sample L-moments in (16) with the population L-monts in (17) and solving the following equations

$$
\ell_{1}=\tau_{1}\left(\hat{\alpha}_{L M}, \hat{\lambda}_{L M}\right) \quad \text { and } \quad \ell_{2}=\tau_{2}\left(\hat{\alpha}_{L M}, \hat{\lambda}_{L M}\right)
$$

\subsection{Ordinary least squares}

Consider a random sample of size $n$ from the APE distribution, and let $x_{1: n}<x_{2: n}<\cdots<x_{n: n}$ be the order observations, then we can obtained the ordinary lease squares (OLS) estimates of $\alpha$ and $\lambda$, denoted by $\hat{\alpha}_{O L S}$ and $\hat{\lambda}_{O L S}$ by minimizing

$$
S(\alpha, \lambda)=\sum_{i=1}^{n}\left\{\frac{\alpha^{\left(1-e^{-\lambda x_{i: n}}\right)}-1}{\alpha-1}-\frac{i}{n+1}\right\}^{2} .
$$

with respect to $\alpha$ and $\lambda$. These estimates can be obtained by solving the following nonlinear equations

$$
\frac{\partial S(\alpha, \lambda)}{\partial \alpha}=\sum_{i=1}^{n}\left\{\frac{\alpha^{\left(1-e^{-\lambda x_{i: n}}\right)}-1}{\alpha-1}-\frac{i}{n+1}\right\} \psi_{1}\left(x_{i: n} \mid \alpha, \lambda\right)=0
$$

and

$$
\frac{\partial S(\alpha, \lambda)}{\partial \lambda}=\sum_{i=1}^{n}\left\{\frac{\alpha^{\left(1-e^{-\lambda x_{i: n}}\right)}-1}{\alpha-1}-\frac{i}{n+1}\right\} \psi_{2}\left(x_{i: n} \mid \alpha, \lambda\right)=0
$$

where

$$
\psi_{1}\left(x_{i: n} \mid \alpha, \lambda\right)=\frac{\alpha^{-e^{-\lambda x_{i: n}}}\left(1-e^{-\lambda x_{i: n}}\right)}{\alpha-1}-\frac{\alpha^{\left(1-e^{-\lambda x_{i: n}}\right)}-1}{(\alpha-1)^{2}}
$$

and

$$
\psi_{2}\left(x_{i: n} \mid \alpha, \lambda\right)=\frac{\log (\alpha)}{\alpha-1} x_{i: n} e^{-\lambda x_{i: n}} \alpha^{\left(1-e^{-\lambda x_{i: n}}\right)} .
$$

\subsection{Weighted least squares}

Using the same notation as in the previous subsection, the weighted least squares (WLS) estimates of $\alpha$ and $\lambda$, denoted by $\hat{\alpha}_{W L S}$ and $\hat{\lambda}_{W L S}$ can be obtained for the APE distribution by minimizing the following function

$$
W(\alpha, \lambda)=\sum_{i=1}^{n} \frac{(n+1)^{2}(n+2)}{i(n-i+1)}\left\{\frac{\alpha^{\left(1-e^{-\lambda x_{i: n}}\right.}-1}{\alpha-1}-\frac{i}{n+1}\right\}^{2},
$$

with respect to $\alpha$ and $\lambda$, or equivalently by solving the following two equations

$$
\frac{\partial W(\alpha, \lambda)}{\partial \alpha}=\sum_{i=1}^{n} \frac{(n+1)^{2}(n+2)}{i(n-i+1)}\left\{\frac{\alpha^{\left(1-e^{-\lambda x_{i: n}}\right)}-1}{\alpha-1}-\frac{i}{n+1}\right\} \psi_{1}\left(x_{i: n} \mid \alpha, \lambda\right)=0
$$

and

$$
\frac{\partial W(\alpha, \lambda)}{\partial \lambda}=\sum_{i=1}^{n} \frac{(n+1)^{2}(n+2)}{i(n-i+1)}\left\{\frac{\alpha^{\left(1-e^{-\lambda x_{i: n}}\right)}-1}{\alpha-1}-\frac{i}{n+1}\right\} \psi_{2}\left(x_{i: n} \mid \alpha, \lambda\right)=0,
$$

where $\psi_{j}\left(x_{i: n} \mid \alpha, \lambda\right), j=1,2$ are defined by (18) and (19). 


\subsection{Method of percentile estimation}

The percentile (PE) method can be used to obtain the PE estimates of $\alpha$ and $\lambda$ which denoted by $\hat{\alpha}_{P E}$ and $\hat{\lambda}_{P E}$ by equating the sample percentile points with the corresponding population percentile points. For the APE distribution and based on the quantile function (7), we can obtain the PE estimates by minimizing the following function with respect to $\alpha$ and $\lambda$

$$
P(\alpha, \lambda)=\sum_{i=1}^{n}\left\{x_{i: n}+\frac{1}{\lambda} \log \left[\frac{\log \left(\frac{\alpha}{1+p_{i}(\alpha-1)}\right)}{\log \alpha}\right]\right\}^{2} .
$$

Also, we can obtain these estimates by solving

$$
\frac{\partial P(\alpha, \lambda)}{\partial \alpha}=\sum_{i=1}^{n}\left\{x_{i: n}+\frac{1}{\lambda} \log \left[\frac{\log \left(\frac{\alpha}{1+p_{i}(\alpha-1)}\right)}{\log \alpha}\right]\right\} \phi_{1}\left(x_{i: n} \mid \alpha, \lambda\right)=0
$$

and

$$
\frac{\partial P(\alpha, \lambda)}{\partial \lambda}=\sum_{i=1}^{n}\left\{x_{i: n}+\frac{1}{\lambda} \log \left[\frac{\log \left(\frac{\alpha}{1+p_{i}(\alpha-1)}\right)}{\log \alpha}\right]\right\} \phi_{2}\left(x_{i: n} \mid \alpha, \lambda\right)=0,
$$

where $p_{i}=\frac{i}{n+1}$ is an estimate of $F\left(x_{i: n} \mid \alpha, \lambda\right)$ and

$$
\phi_{1}\left(x_{i: n} \mid \alpha, \lambda\right)=\frac{1}{\lambda}\left\{\frac{\frac{1}{\alpha}-\frac{p_{i}}{1+p_{i}(\alpha-1)}}{\log \alpha-\log \left(1+p_{i}(\alpha-1)\right)}-\frac{1}{\alpha \log \alpha}\right\}
$$

and

$$
\phi_{2}\left(x_{i: n} \mid \alpha, \lambda\right)=\log \left\{\frac{\log \left(\frac{\alpha}{1+p_{i}(\alpha-1)}\right)}{\log \alpha}\right\} .
$$

\subsection{Maximum product of spacing method}

The maximum product of spacing (MPS) method was introduced by Cheng and Amin $(1979,1983)$ as an alternative to the maximum likelihood method. They proved that the MPS estimates are efficient as the MLEs. For the APE distribution, we define the uniform spacings of a random sample of size $n$ as

$$
D_{i}(\alpha, \lambda)=F\left(x_{i: n} \mid \alpha, \lambda\right)-F\left(x_{i-1: n} \mid \alpha, \lambda\right), i=1,2, \ldots, n,
$$

where $F\left(x_{0: n} \mid \alpha, \lambda\right)=0, F\left(x_{n+1: n} \mid \alpha, \lambda\right)=1$. Now, the MPS estimates of $\alpha$ and $\lambda$ are denoted by $\hat{\alpha}_{M P S}$ and $\hat{\lambda}_{M P S}$ can be obtained by maximizing the following function with respect to $\alpha$ and $\lambda$

$$
M(\alpha, \lambda)=\frac{1}{n+1} \sum_{i=1}^{n+1} \log D_{i}(\alpha, \lambda)
$$

The $\hat{\alpha}_{M P S}$ and $\hat{\lambda}_{M P S}$ can also be obtained by solving

$$
\frac{\partial M(\alpha, \lambda)}{\partial \alpha}=\frac{1}{n+1} \sum_{i=1}^{n+1} \frac{\psi_{1}\left(x_{i: n} \mid \alpha, \lambda\right)-\psi_{1}\left(x_{i-1: n} \mid \alpha, \lambda\right)}{D_{i}(\alpha, \lambda)}=0
$$

and

$$
\frac{\partial M(\alpha, \lambda)}{\partial \lambda}=\frac{1}{n+1} \sum_{i=1}^{n+1} \frac{\psi_{2}\left(x_{i: n} \mid \alpha, \lambda\right)-\psi_{2}\left(x_{i-1: n} \mid \alpha, \lambda\right)}{D_{i}(\alpha, \lambda)}=0,
$$

where $\psi_{j}\left(x_{i: n} \mid \alpha, \lambda\right), j=1,2$ are give by (18) and (19). 


\subsection{Minimum distance methods}

In this subsection, we use two estimation methods based on the minimization of two well-known goodness-of-fit statistics. The two methods are Cramér-von-Mises method and method of Anderson-Darling which based on the difference between the estimates of the CDF and the empirical distribution function, see D'Agostino (1986).

\subsubsection{Cramér-von-Mises}

The Cramér-von-Mises (CM) estimates $\hat{\alpha}_{C M}$ and $\hat{\lambda}_{C M}$ of the APE distribution parameters $\alpha$ and $\lambda$ are obtained by minimizing the following function with respect to $\alpha$ and $\lambda$

$$
C(\alpha, \lambda)=\frac{1}{12 n}+\frac{1}{n} \sum_{i=1}^{n}\left\{\frac{\alpha^{\left(1-e^{-\lambda x_{i: n}}\right)}-1}{\alpha-1}-\frac{2 i-1}{2 n}\right\}^{2}
$$

These estimates also can be obtained by solving

$$
\frac{\partial C(\alpha, \lambda)}{\partial \alpha}=\sum_{i=1}^{n}\left\{\frac{\alpha^{\left(1-e^{-\lambda x_{i: n}}\right)}-1}{\alpha-1}-\frac{2 i-1}{2 n}\right\} \psi_{1}\left(x_{i: n} \mid \alpha, \lambda\right)=0
$$

and

$$
\frac{\partial C(\alpha, \lambda)}{\partial \lambda}=\sum_{i=1}^{n}\left\{\frac{\alpha^{\left(1-e^{-\lambda x_{i: n}}\right)}-1}{\alpha-1}-\frac{2 i-1}{2 n}\right\} \psi_{2}\left(x_{i: n} \mid \alpha, \lambda\right)=0 .
$$

where $\psi_{j}\left(x_{i: n} \mid \alpha, \lambda\right), j=1,2$ are given by (18) and (19).

\subsubsection{Method of Anderson-Darling}

The Anderson-Darling (AD) estimates of the APE distribution parameters $\alpha$ and $\lambda$ denoted by $\hat{\alpha}_{A D}$ and $\hat{\lambda}_{A D}$ can be obtained by minimizing the following function

$$
A(\alpha, \lambda)=-n-\frac{1}{n} \sum_{i=1}^{n}(2 i-1)\left\{\log F\left(x_{i: n} \mid \alpha, \lambda\right)+\log \bar{F}\left(x_{n-i+1: n} \mid \alpha, \lambda\right)\right\},
$$

with respect to $\alpha$ and $\lambda$, or by solving the following two equations

$$
\frac{\partial A(\alpha, \lambda)}{\partial \alpha}=\sum_{i=1}^{n}(2 i-1)\left\{\frac{\psi_{1}\left(x_{i: n} \mid \alpha, \lambda\right)}{F\left(x_{i: n} \mid \alpha, \lambda\right)}-\frac{\psi_{1}\left(x_{n-i+1: n} \mid \alpha, \lambda\right)}{\bar{F}\left(x_{n-i+1: n} \mid \alpha, \lambda\right)}\right\}=0
$$

and

$$
\frac{\partial A(\alpha, \lambda)}{\partial \lambda}=\sum_{i=1}^{n}(2 i-1)\left\{\frac{\psi_{2}\left(x_{i: n} \mid \alpha, \lambda\right)}{F\left(x_{i: n} \mid \alpha, \lambda\right)}-\frac{\psi_{2}\left(x_{n-i+1: n} \mid \alpha, \lambda\right)}{\bar{F}\left(x_{n-i+1: n} \mid \alpha, \lambda\right)}\right\}=0 .
$$

where $\psi_{j}(. \mid \alpha, \lambda), j=1,2$ are defined by (18) and (19).

\section{Simulation Study}

Here, we conduct Monte Carlo simulation studies in order to assess the performance among of the different estimation methods introduced and discussed in this article. The application of simulation studies involve the following steps:

1. Generate random samples of size $n$ from ALE distribution

2. Calculate $\hat{\alpha}$ and $\hat{\lambda}$ via 1-MLE, 2-OLS, 3-WLS, 4-PE, 5-MPS, 6-CM, 7-AD

3. Repeat previous steps $\mathrm{N}$ times 
4. Set $\boldsymbol{\theta}=(\alpha, \lambda)^{T}$ and $\widehat{\boldsymbol{\theta}}=(\widehat{\alpha}, \widehat{\lambda})$, compute the mean relative estimates (MREs) $\sum_{i=1}^{N} \frac{\hat{\theta}_{\ell, i} / \theta_{\ell}}{N}$ for $\ell=1,2$ and the root squared of the mean squared errors (RMSEs) $\sqrt{\sum_{i=1}^{N} \frac{\left(\hat{\theta}_{\ell, i}-\theta_{\ell}\right)^{2}}{N}}$.

By using this approach, the most efficient estimation method is expected to have MREs approaching to 1 and RMSEs decaying to 0 , as the sample size getting large. The results of the Monte Carlo simulation procedures are obtained from simulating 10, 000 samples from $\operatorname{APE}(\alpha, \lambda)$ distribution of sizes $n=10,20,30, \ldots, 250$ and $(\alpha, \lambda)=(0.5,0.5)$, $(3,3),(10,0.3),(0.5,3),(5,1),(5,15)$. The estimates of the parameters are obtained by executing the $\mathrm{n} l \mathrm{~s}$ function implemented in the software R. It should be noted that the estimation methods are put in the same conditions, i.e., using the same sample sizes and same initial values. However, some estimation methods namely method of moments and L-moments do not preform well in finding the parameter estimates and therefore they are excluded from the simulation study. Additionally, a rate convergence study is performed to assess the behavior of the convergence rate of the numerical solution. The rate study is carried out by calculating the rate of times that each estimation fails in finding the numerical solution. Figure 2 highlights the proportion failure of each method. It can be seen from this figure that the MLEs, MPS and the CM estimates are succeed in locating the numerical solution for a large number of samples whereas the other methods are failed. Figures 3-8 demonstrate the RMSEs and MREs of $\widehat{\alpha}$ and $\widehat{\lambda}$ using the MLEs, MPS, CM based on 10, 000 replicated samples of sizes $n=10,20, \ldots, 250$. It is evident that for large sample size, the calculated RMSEs using these estimators tend to zero and the values of MREs tend to one. This implies that these estimators are consistent and asymptotically unbiased. Indeed, consistent estimates using these estimators can be achieved for $n>50$.

The MPS estimates of $\alpha$ behave better than the MLEs and CM estimates for $n>50$ in terms of MREs. For the case $\lambda$, the MLEs and CM estimates behave slightly better than MPS for small sample sizes. However, the MPS estimates have the smallest RMSEs. On the other hand, the MPS estimates and MLEs have the lowest rate of convergence for different values of $\alpha$ and $\lambda$. Taking into account all the results with the attractive properties of MPS such as invariance, consistency, and asymptotic normality, we conclude that the MPS estimators are strongly competitive method compared to the ML method for estimating parameters of APE distribution.
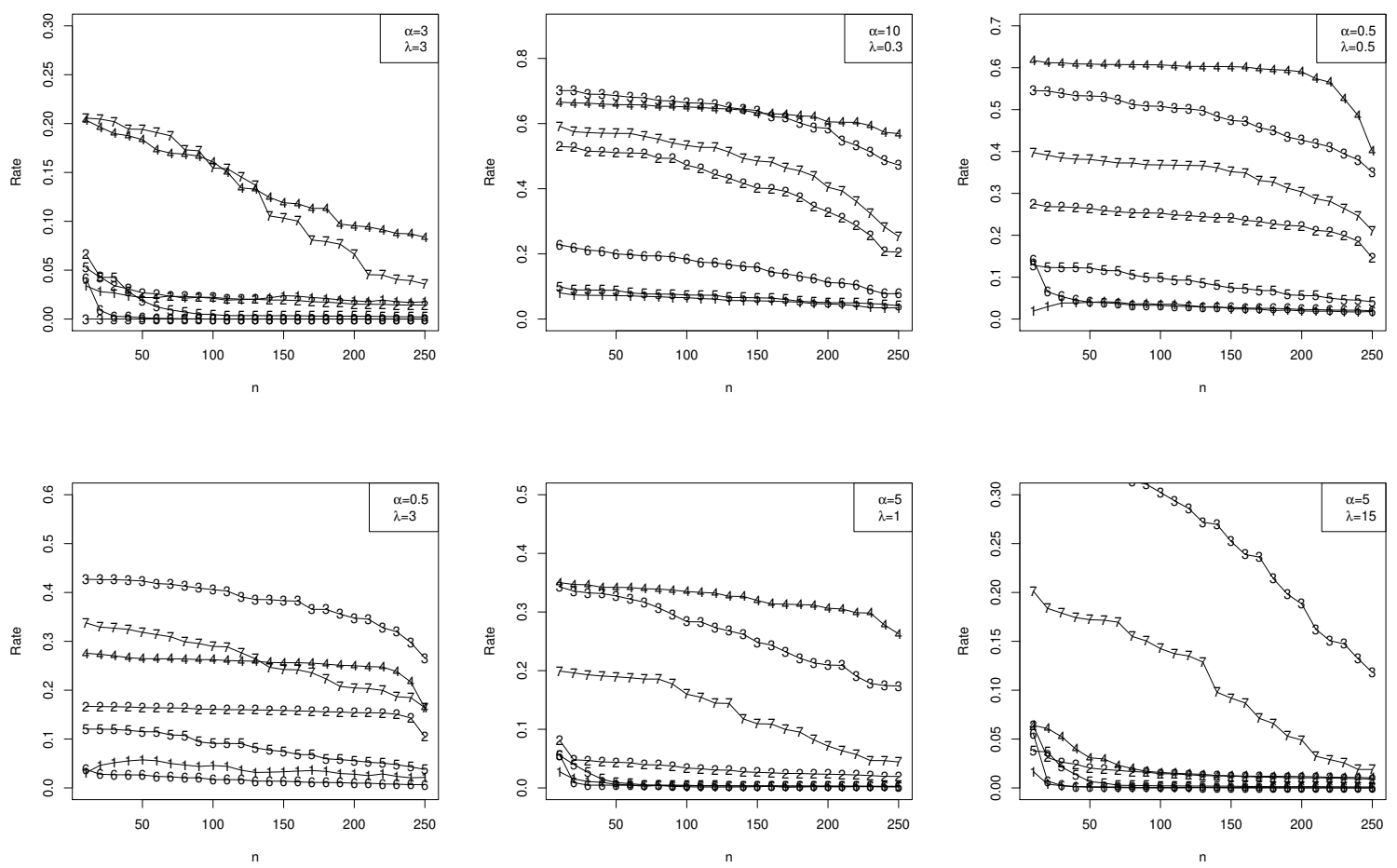

Figure 2: Rate of convergence considering different values of $\boldsymbol{\theta}$ and different estimation procedures: 1-MLE, 2-OLS, 3-WLS, 4-PE, 5-MPS, 6-CM, 7-AD 

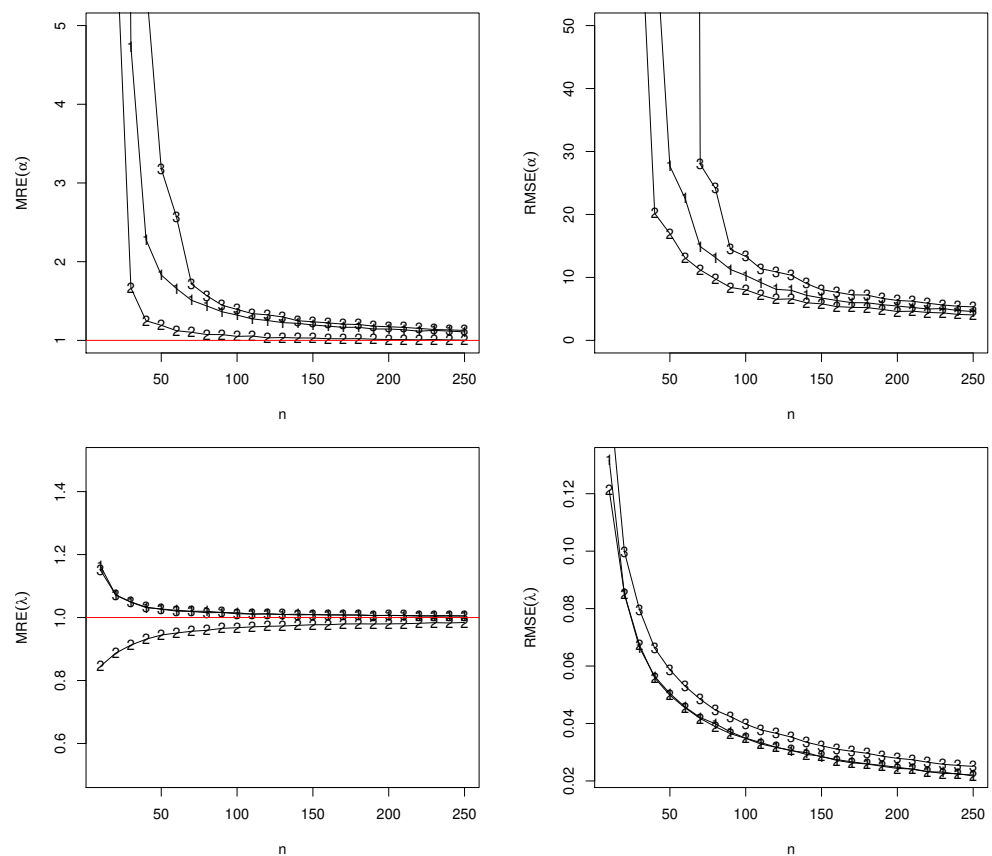

Figure 3: RMSEs and MREs of various estimators when $\alpha=10$ and $\lambda=0.3$ based on 10,000 replications using the estimation methods 1-MLE, 2-MPS, 3-CM
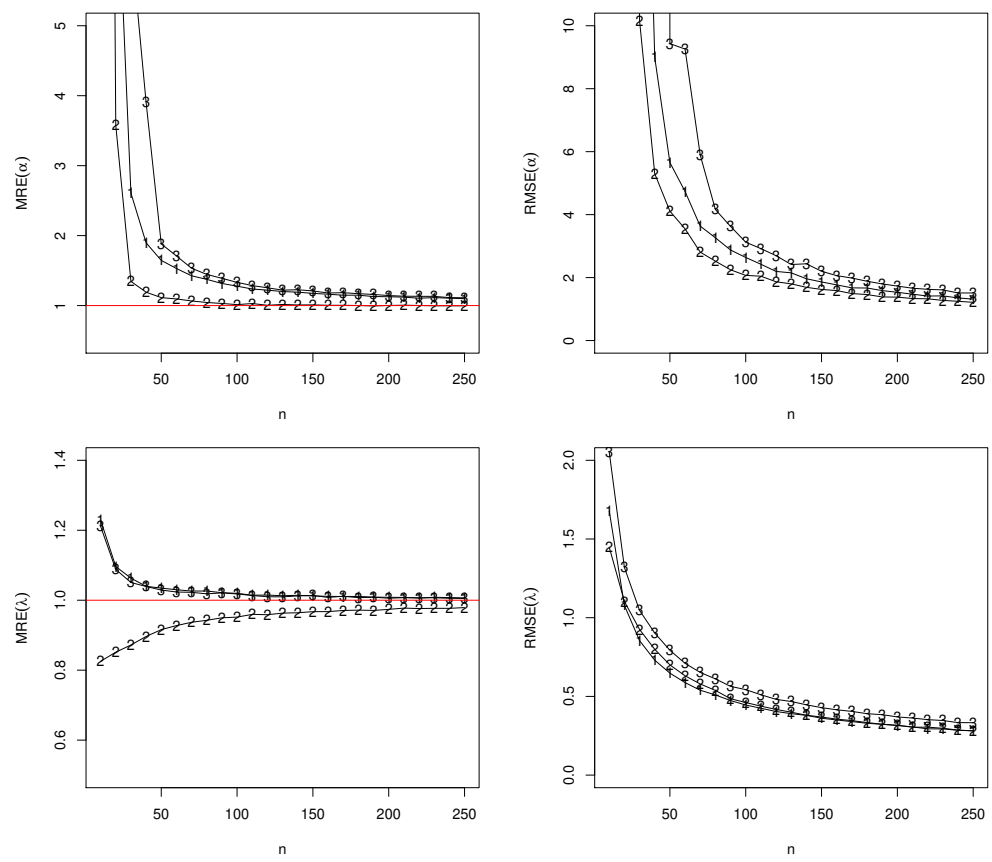

Figure 4: RMSEs and MREs of various estimators when $\alpha=3$ and $\lambda=3$ based on 10,000 replications using the estimation methods 1-MLE, 2-MPS, 3-CM 

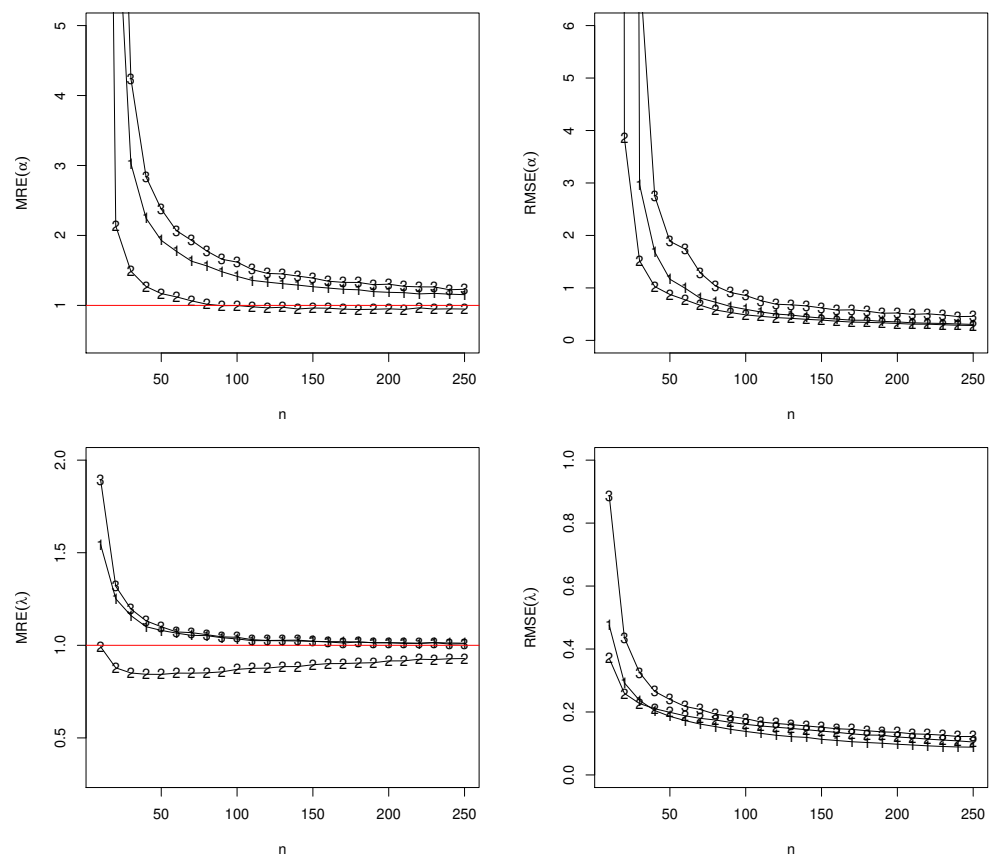

Figure 5: RMSEs and MREs of various estimators when $\alpha=0.5$ and $\lambda=0.5$ based on 10,000 replications using the estimation methods 1-MLE, 2-MPS, 3-CM
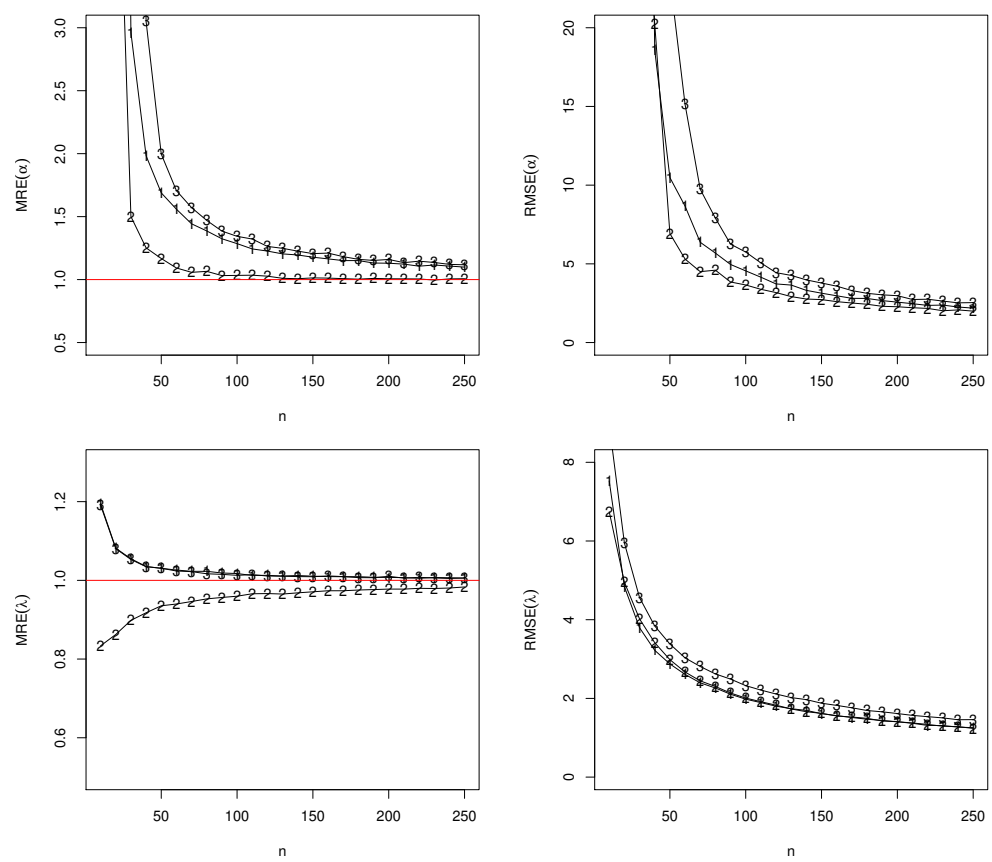

Figure 6: RMSEs and MREs of various estimators when $\alpha=5$ and $\lambda=15$ based on 10,000 replications using the estimation methods 1-MLE, 2-MPS, 3-CM 

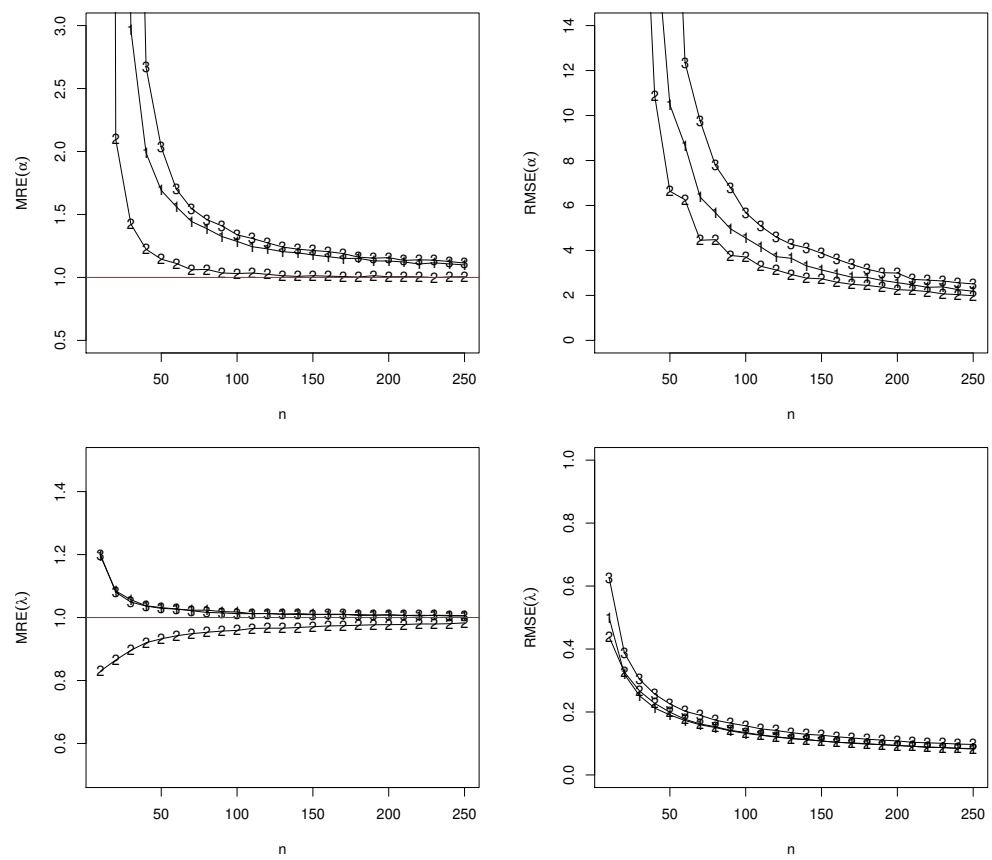

Figure 7: RMSEs and MREs of various estimators when $\alpha=5$ and $\lambda=1$ based on 10,000 replications using the estimation methods 1-MLE, 2-MPS, 3-CM
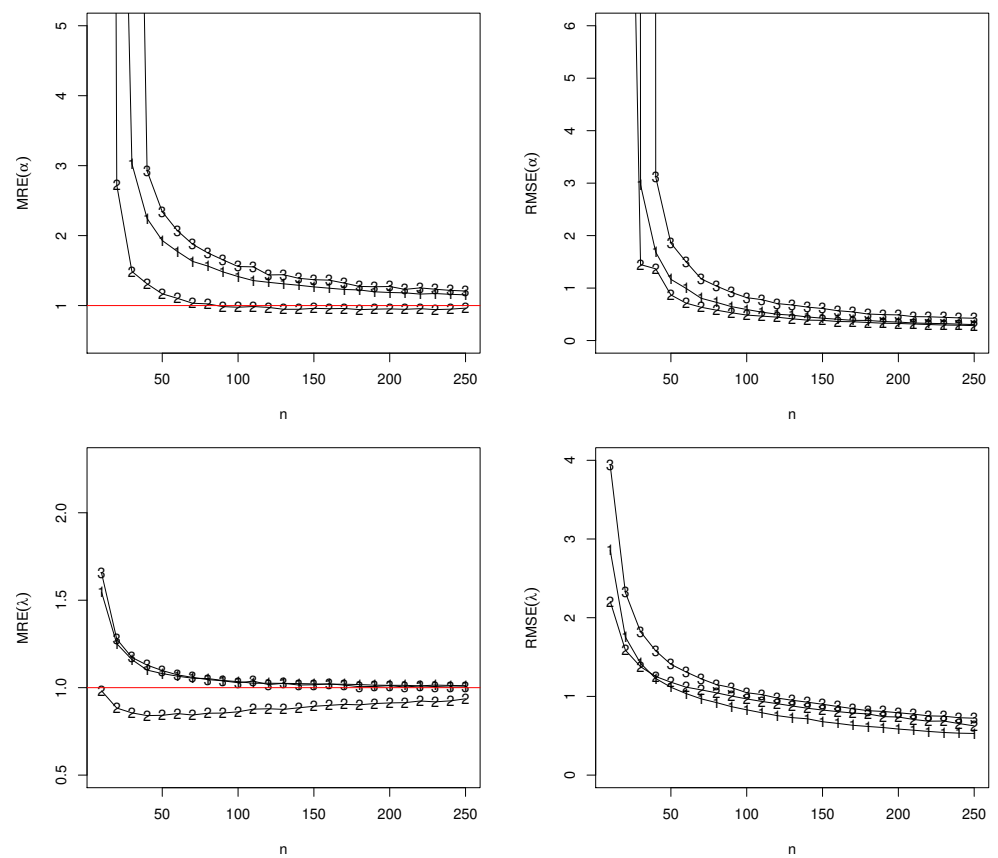

Figure 8: RMSEs and MREs of various estimators when $\alpha=0.5$ and $\lambda=3$ based on 10,000 replications using the estimation methods 1-MLE, 2-MPS, 3-CM 


\section{Real Data Analysis}

In this section, we illustrate the practical importance of the APE model by analyzing two real data sets. The first data set represents failures times of 50 items from Murthy et al. (2004). The second data represent the survival times of 121 patients with breast cancer obtained from a large hospital in a period from 1929 to 1938 (Lee, 1992).

The APE distribution is compared with many well-known rival lifetime distributions for both data sets. The rival models namely, transmuted generalized exponential (TGE) (Khan et al., 2017), Marshall-Olkin logistic-exponential (MOLE) (Mansoor et al., 2018), alpha logarithmic transformed Weibull (ALTW) (Nassar et al., 2018a), MarshallOlkin exponential (MOE) (Marshall and Olkin, 1997), Lindley Weibull (Cordeiro et al., 2018), generalized odd loglogistic exponential (GOLLE) (Afify et al., 2019), exponentiated exponential (EE) (Gupta and Kundu, 2001), beta exponential (BE) (Jones, 2004 and Nadarajah and Kotz,2006), gamma (Ga), Weibull (W) (Weibull, 1951) and exponential (E) distributions. The PDFs of these models are given (for $x>0$ ) by

TGE $: f(x)=\alpha \lambda e^{-\lambda x}\left(1-e^{-\lambda x}\right)^{\alpha-1}\left[1+\theta-2 \theta\left(1-e^{-\lambda x}\right)^{\alpha}\right], \alpha, \lambda>0,|\theta| \leq 1$.

MOLE : $f(x)=\alpha \theta \lambda e^{\lambda x}\left(e^{\lambda x}-1\right)^{-\alpha-1}\left[1+\theta\left(e^{\lambda x}-1\right)^{-\alpha}\right]^{-2}, \alpha, \lambda, \theta>0$.

ALTW $: f(x)=\frac{(\alpha-1) \lambda \beta x^{\beta-1} e^{-\lambda x^{\beta}}}{\log (\alpha)\left[\alpha-(\alpha-1)\left(1-e^{-\lambda x^{\beta}}\right)\right]}, \alpha, \lambda, \beta>0, \alpha \neq 1$.

MOE $: f(x)=\alpha \lambda e^{-\lambda x}\left[1-(1-\alpha) e^{-\lambda x}\right]^{-2}, \alpha, \lambda>0$.

$\mathrm{LW}: f(x)=\frac{\beta \theta^{2}}{\theta+1}\left[\alpha^{\beta} x^{\beta-1}+\alpha^{2 \beta} x^{2 \beta-1}\right] e^{-\theta(\alpha x)^{\beta}}, \alpha, \lambda, \theta>0$.

GOLLE $: f(x)=\frac{\alpha \theta \lambda e^{-\lambda x}\left(1-e^{-\lambda x}\right)^{\alpha \theta-1}\left[1-\left(1-e^{-\lambda x}\right)^{\theta}\right]^{\alpha-1}}{\left\{\left(1-e^{-\lambda x}\right)^{\alpha \theta}+\left[1-\left(1-e^{-\lambda x}\right)^{\theta}\right]^{\alpha}\right\}^{2}}, \alpha, \lambda, \theta>0$.

$\operatorname{EEx}: f(x)=\alpha \lambda e^{-\lambda x}\left(1-e^{-\lambda x}\right)^{\alpha-1}, \alpha, \lambda>0$.

$\mathrm{BE}: f(x)=\frac{\lambda}{B(a, b)} e^{-\lambda b x}\left(1-e^{-\lambda x}\right)^{a-1}, a, b, \lambda>0$.

$\mathrm{Ga}: f(x)=\frac{b^{-a}}{\Gamma(a)} x^{a-1} e^{-x / b}, a, b>0$.

$\mathrm{W}: f(x)=\beta \lambda^{\beta} x^{\beta-1} e^{-(\lambda x)^{\beta}}, \alpha, \beta>0$.

The Kolmogorov-Smirnov (KS) test and its p-value (PV) are considered to check the goodness of fit. Tables 1 and 2 provide the KS test and PV for all fitted distributions. Both criteria provide evidence in favor of the APE distribution for both data sets. Hence, the APE distribution can be seen as a competitive distribution of practical interest for different fields. Figures 9 and 10 show the fitted PDF, CDF, survival function (SF) and PP plots of the APE distribution for both data sets, respectively. 
Table 1: The estimates (SEs in parentheses), KS and its PV for failures times data

\begin{tabular}{|c|c|c|c|c|c|}
\hline \multirow{2}{*}{$\begin{array}{l}\text { Distribution } \\
\operatorname{APE}(\alpha, \lambda)\end{array}$} & \multicolumn{3}{|c|}{ Estimates } & \multirow{2}{*}{$\begin{array}{c}\text { KS } \\
0.0898\end{array}$} & \multirow{2}{*}{$\begin{array}{l}\text { PV } \\
0.8144\end{array}$} \\
\hline & $\begin{array}{l}0.24232 \\
(0.3629)\end{array}$ & $\begin{array}{l}0.08755 \\
(0.0418)\end{array}$ & & & \\
\hline $\operatorname{TGE}(\alpha, \lambda, \theta)$ & $\begin{array}{l}0.60962 \\
(0.2165)\end{array}$ & $\begin{array}{l}0.10672 \\
(0.0234)\end{array}$ & $\begin{array}{l}-0.29237 \\
(0.6050)\end{array}$ & 0.0905 & 0.8068 \\
\hline $\operatorname{MOLE}(\lambda, \alpha, \theta)$ & $\begin{array}{l}0.17497 \\
(0.0777)\end{array}$ & $\begin{array}{l}0.71217 \\
(0.1524)\end{array}$ & $\begin{array}{l}1.22084 \\
(0.6718)\end{array}$ & 0.0912 & 0.7994 \\
\hline $\operatorname{ALTW}(\alpha, \beta, \lambda)$ & $\begin{array}{l}13.6774 \\
(34.949) \\
\end{array}$ & $\begin{array}{l}0.64048 \\
(0.1408)\end{array}$ & $\begin{array}{l}0.56971 \\
(0.4752) \\
\end{array}$ & 0.0921 & 7897 \\
\hline $\operatorname{MOE}(\alpha, \lambda)$ & $\begin{array}{l}0.5023 \\
(0.2756)\end{array}$ & $\begin{array}{l}0.0892 \\
(0.0326)\end{array}$ & & 0.0988 & 0.7124 \\
\hline $\mathrm{LW}(a, \beta, \theta)$ & $\begin{array}{l}0.27895 \\
(0.7200)\end{array}$ & $\begin{array}{l}0.71929 \\
(0.1329)\end{array}$ & $\begin{array}{l}0.96995 \\
(1.4528)\end{array}$ & 0.1018 & 0.6778 \\
\hline $\operatorname{GOLLE}(\alpha, \theta, \lambda)$ & $\begin{array}{l}1.02903 \\
(0.6426)\end{array}$ & $\begin{array}{l}0.66277 \\
(0.5163) \\
\end{array}$ & $\begin{array}{l}0.09479 \\
(0.0960)\end{array}$ & 0.1031 & 0.6627 \\
\hline $\mathrm{EE}(\alpha$, & $\begin{array}{l}0.68630 \\
(0.1193)\end{array}$ & $\begin{array}{l}0.09915 \\
(0.0204)\end{array}$ & & 0.1032 & 0.6607 \\
\hline $\mathrm{BE}(a, b, \lambda)$ & $\begin{array}{l}0.69490 \\
(0.1185)\end{array}$ & $\begin{array}{l}5.39695 \\
(10.814)\end{array}$ & $\begin{array}{l}0.01691 \\
(0.0345)\end{array}$ & 0.1050 & 0.6396 \\
\hline $\mathrm{Ga}(a, b)$ & $\begin{array}{l}0.69521 \\
(0.1183)\end{array}$ & $\begin{array}{l}0.08889 \\
(0.0213)\end{array}$ & & 0.1050 & 0.6389 \\
\hline $\mathrm{W}(\beta, \lambda)$ & $\begin{array}{l}0.80021 \\
(0.0906)\end{array}$ & $\begin{array}{l}0.14349 \\
(0.0266)\end{array}$ & & 0.1118 & 0.5590 \\
\hline $\mathrm{E}(\lambda)$ & $\begin{array}{l}0.12786 \\
(0.0180)\end{array}$ & & & 0.1089 & 0.5934 \\
\hline
\end{tabular}
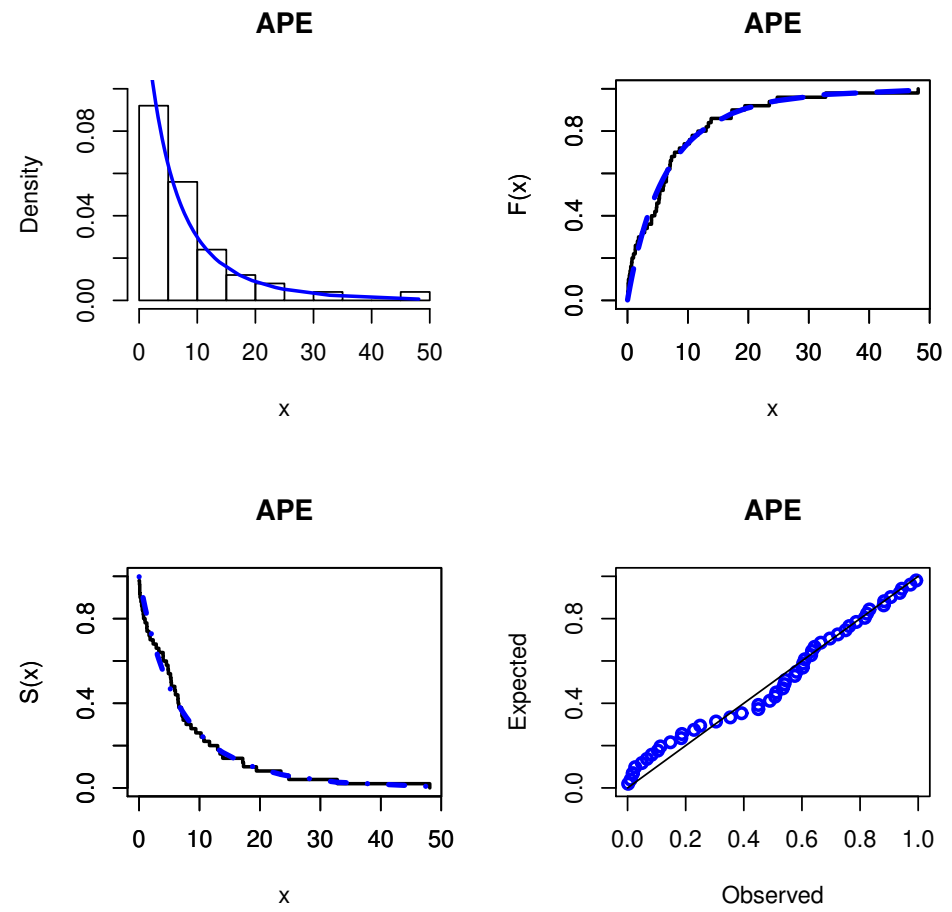

Figure 9: Fitted PDF, CDF, SF and PP plots of the APE distribution for failures times dat. 
Table 2: The estimates (SEs in parentheses), KS and its PV for survival times data

\begin{tabular}{|c|c|c|c|c|c|}
\hline \multirow{2}{*}{$\begin{array}{l}\text { Distribution } \\
\operatorname{APE}(\alpha, \lambda)\end{array}$} & \multicolumn{3}{|c|}{ Estimates } & \multirow{2}{*}{$\frac{\mathrm{KS}}{0.0534}$} & \multirow{2}{*}{$\frac{\mathrm{PV}}{0.8802}$} \\
\hline & $\begin{array}{l}7.62528 \\
(4.1780)\end{array}$ & $\begin{array}{l}0.03314 \\
(0.0037)\end{array}$ & & & \\
\hline $\operatorname{MOLE}(\lambda, \alpha, \theta)$ & $\begin{array}{l}0.02855 \\
(0.0092)\end{array}$ & $\begin{array}{l}1.13195 \\
(0.1874)\end{array}$ & $\begin{array}{l}2.15622 \\
(1.1819)\end{array}$ & 0.0562 & 0.8383 \\
\hline $\operatorname{ALTW}(\alpha, \beta, \lambda)$ & $\begin{array}{l}1.14680 \\
(0.7694)\end{array}$ & $\begin{array}{l}1.28656 \\
(0.0760)\end{array}$ & $\begin{array}{l}0.00676 \\
(0.0027)\end{array}$ & 0.0585 & 0.8013 \\
\hline $\operatorname{MOE}(\alpha, \lambda)$ & $\begin{array}{l}0.0199 \\
(0.0014)\end{array}$ & $\begin{array}{l}1.3056 \\
(0.0934)\end{array}$ & & 0.0598 & 7783 \\
\hline $\mathrm{W}(\beta, \lambda)$ & $\begin{array}{l}1.30564 \\
(0.0934)\end{array}$ & $\begin{array}{l}0.01994 \\
(0.0014)\end{array}$ & & 0.0598 & 7783 \\
\hline $\mathrm{LW}(\alpha, \beta, \theta)$ & $\begin{array}{l}0.19516 \\
(0.3502)\end{array}$ & $\begin{array}{l}0.98582 \\
(0.1344)\end{array}$ & $\begin{array}{l}0.20926 \\
(0.2874)\end{array}$ & 0.0606 & 0.7655 \\
\hline $\operatorname{TGE}(\alpha, \lambda, \theta)$ & $\begin{array}{l}0.97441 \\
(0.2407)\end{array}$ & $\begin{array}{l}0.02977 \\
(0.0032)\end{array}$ & $\begin{array}{l}-0.80218 \\
(0.2343)\end{array}$ & 0.0687 & 0.6168 \\
\hline $\operatorname{GOLLE}(\alpha, \theta, \lambda)$ & $\begin{array}{l}1.34099 \\
(0.5115)\end{array}$ & $\begin{array}{l}0.98401 \\
(0.5444)\end{array}$ & $\begin{array}{l}0.01866 \\
(0.0105)\end{array}$ & 0.0759 & 0.4880 \\
\hline $\mathrm{Ga}(a, b)$ & $\begin{array}{l}1.49614 \\
(0.1748)\end{array}$ & $\begin{array}{l}0.03229 \\
(0.0044)\end{array}$ & & 0.0762 & 0.4832 \\
\hline$\overline{\mathrm{BE}}(a, b, \lambda)$ & $\begin{array}{l}1.49787 \\
(0.1764)\end{array}$ & $\begin{array}{l}4.31185 \\
(1.7242)\end{array}$ & $\begin{array}{l}0.00712 \\
(0.0026)\end{array}$ & 0.0767 & 0.4746 \\
\hline $\mathrm{EE}(\alpha, \lambda)$ & $\begin{array}{l}1.51682 \\
(0.1926)\end{array}$ & $\begin{array}{l}0.02774 \\
(0.0029)\end{array}$ & & 0.0804 & 0.4134 \\
\hline $\mathrm{E}(\lambda)$ & $\begin{array}{l}0.02158 \\
(0.0019)\end{array}$ & & & 0.1203 & 0.0602 \\
\hline
\end{tabular}
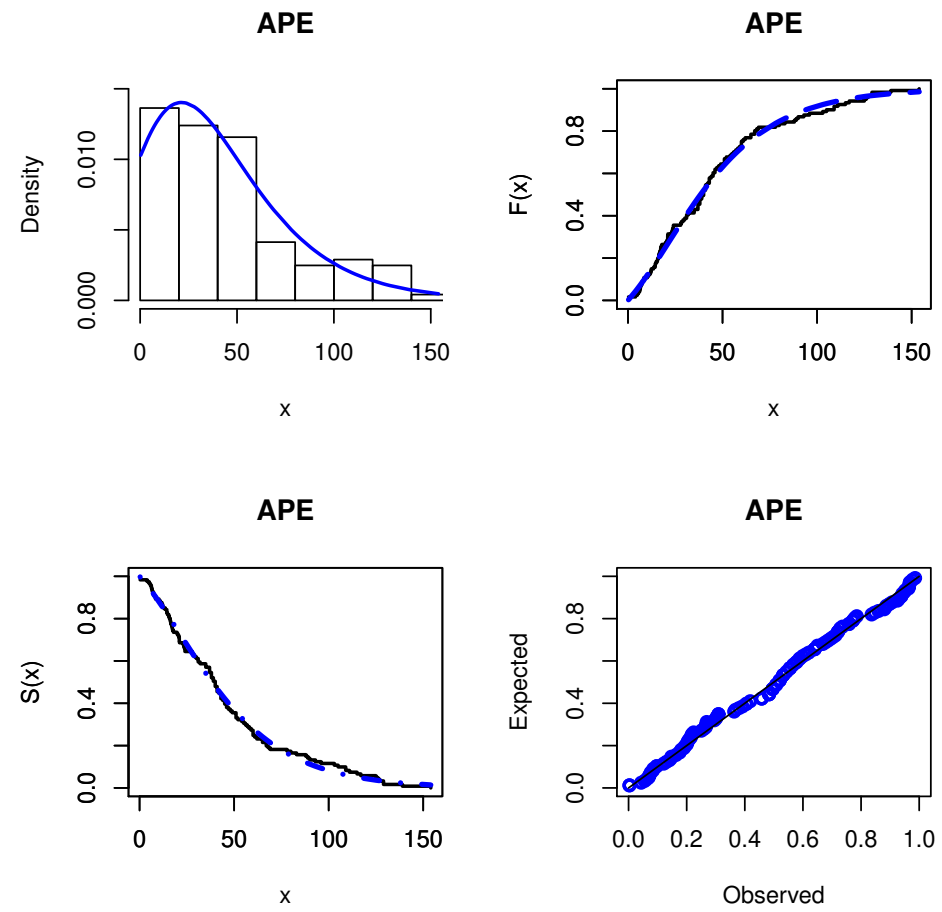

Figure 10: Fitted PDF, CDF, SF and PP plots of the APE distribution for survival times data. 
Tables 3 and 4 provide the estimates of the unknown parameters of the APE distribution, for both the data sets, using seven methods of estimation and the values of $-\ell, \mathrm{KS}$ and corresponding P-values. The WLS method can be used to estimate the parameters of the APE distribution for data I, whereas, the MLE method can be used to estimate the parameters of the APE distribution for data II. However, all estimation methods perform well.

Table 3: The parameter estimates under various methods and the goodness of fit statistics for failures times data.

\begin{tabular}{cccccc}
\hline \hline Method & $\widehat{\alpha}$ & $\widehat{\lambda}$ & $-\widehat{\ell}$ & $\mathrm{K}-\mathrm{S}$ & $\mathrm{p}$-value \\
\hline MLE & 0.2426 & 0.0876 & 152.028 & 0.0898 & 0.8145 \\
OLS & 0.2351 & 0.0831 & 152.063 & 0.0910 & 0.8025 \\
WLS & 0.1288 & 0.0690 & 152.122 & 0.088 & 0.8359 \\
PEs & 0.0182 & 0.0381 & 152.351 & 0.0917 & 0.7945 \\
MPS & 0.0133 & 0.0352 & 152.353 & 0.0926 & 0.7849 \\
CMEs & 0.3618 & 0.0971 & 152.075 & 0.0924 & 0.7863 \\
ADEs & 0.1649 & 0.0773 & 152.129 & 0.0942 & 0.7669 \\
\hline
\end{tabular}

Table 4: The parameter estimates under various methods and the goodness of fit statistics for survival times data.

\begin{tabular}{cccccc}
\hline \hline Method & $\widehat{\alpha}$ & $\widehat{\lambda}$ & $-\widehat{\ell}$ & $\mathrm{K}-\mathrm{S}$ & $\mathrm{p}$-value \\
\hline MLE & 7.6038 & 0.0331 & 579.155 & 0.0534 & 0.8802 \\
OLS & 6.3464 & 0.0321 & 579.210 & 0.0582 & 0.8071 \\
WLS & 6.7606 & 0.0323 & 579.181 & 0.0550 & 0.8581 \\
PEs & 6.8468 & 0.0321 & 579.194 & 0.0505 & 0.917 \\
MPS & 3.2587 & 0.0275 & 580.432 & 0.0693 & 0.6059 \\
CMEs & 6.9080 & 0.0327 & 579.170 & 0.0579 & 0.8121 \\
ADEs & 6.6371 & 0.0322 & 579.191 & 0.0548 & 0.8599 \\
\hline
\end{tabular}

\section{Competing Risks Analysis}

In reliability and medical studies, the cause of death of units can be due to more than one source. This problem is known in the literature as the analysis of competing risks model. In this section, we consider the APE distribution to the competing risks data. We derive the MLEs of the APE distribution parameters with different parameters. We also analyze one real data set to show the flexibility of the APE distribution in modelling competing risks data.

\subsection{Estimation under competing risks data}

Consider a lifetime experiment with $n$ identical objects. Without loss of generality, we assume that the failure of the objects due to only two causes of failure. Let $X_{1 i}$ and $X_{2 i}$ are the latent failure time of the $i-t h$ object, where $i=1,2, \ldots, n$, then $X_{i}=\min \left\{X_{1 i}, X_{2 i}\right\}$. Based on the competing risks data we have the following observations

$$
\left(x_{1}, \delta_{1}\right),\left(x_{2}, \delta_{2}\right), \ldots,\left(x_{n}, \delta_{n}\right)
$$

where $\delta_{i} \in(1,2)$ is an indicator to determine the cause of failure of the $i t h$ unit. Furthermore, let

$$
I\left(\delta_{i}=1\right)=\left\{\begin{array}{ll}
1, & \delta_{i}=1 \\
0 & \text { else }
\end{array} \quad \text { and } I\left(\delta_{i}=2\right)= \begin{cases}1, & \delta_{i}=2 \\
0 & \text { else }\end{cases}\right.
$$

then, the random variables $n_{1}=\sum_{i=1}^{n} I\left(\delta_{i}=1\right)$ and $n_{2}=\sum_{i=1}^{n} I\left(\delta_{i}=2\right)$ are the number of failures due to cause one and cause two, respectively, and $n=n_{1}+n_{2}$. Now, for a random sample of size $n$ we can write the likelihood function of the observed data $\left(x_{1}, \delta_{1}\right), \ldots,\left(x_{n}, \delta_{n}\right)$ as follows

$$
L=\prod_{i=1}^{n}\left\{\left[f_{1}\left(x_{i}\right) \bar{F}_{2}\left(x_{i}\right)\right]^{I\left(\delta_{i}=1\right)}\left[f_{2}\left(x_{i}\right) \bar{F}_{1}\left(x_{i}\right)\right]^{I\left(\delta_{i}=2\right)}\right\}
$$


where $\bar{F}_{k}=1-F_{k}(x), k=1,2$. Now, let $X_{k i}^{\prime} s, k=1,2$ are APE random variables with parameters $\alpha_{k}$ and $\lambda_{k}$ with the following PDF

$$
f_{k}(x)=\frac{\lambda_{k} \log \alpha_{k}}{\alpha_{k}-1} e^{-\lambda_{k} x} \alpha_{k}^{\left(1-e^{-\lambda_{k} x}\right)}, k=1,2 \alpha_{k} \neq 1
$$

and survival function in the form

$$
\bar{F}_{k}(x)=\frac{\alpha_{k}}{\alpha_{k}-1}\left(1-\alpha_{k}^{-e^{-\lambda_{k} x}}\right), k=1,2, \alpha_{k} \neq 1 .
$$

Then from (20), (21) and (22) we can obtain the likelihood function as follows

$$
\begin{aligned}
L & =\left[\frac{\alpha_{1} \alpha_{2}}{\left(\alpha_{1}-1\right)\left(\alpha_{2}-1\right)}\right]^{n}\left(\lambda_{1} \log \alpha_{1}\right)^{n_{1}}\left(\lambda_{2} \log \alpha_{2}\right)^{n_{2}} e^{-\left(\lambda_{1}+\lambda 2\right) \sum_{i=1}^{n} x_{i}} \alpha_{1}^{-\sum_{i=1}^{n_{1}} e^{-\lambda_{1} x_{i}}} \\
& \times \alpha_{2}^{-\sum_{i=1}^{n_{2}} e^{-\lambda_{2} x_{i}}} \exp \left\{\sum_{i=1}^{n_{1}} \log \left(1-\alpha_{1}^{-e^{-\lambda_{1} x_{i}}}\right)+\sum_{i=1}^{n_{2}} \log \left(1-\alpha_{2}^{-e^{-\lambda_{2} x_{i}}}\right)\right\} .
\end{aligned}
$$

The log-likelihood function of (23) can be written in the following form

$$
\begin{aligned}
\log L & =n \log \left[\frac{\alpha_{1} \alpha_{2}}{\left(\alpha_{1}-1\right)\left(\alpha_{2}-1\right)}\right]+n_{1} \log \left(\lambda_{1} \log \alpha_{1}\right)+n_{2} \log \left(\lambda_{2} \log \alpha_{2}\right) \\
& -\left(\lambda_{1}+\lambda 2\right) \sum_{i=1}^{n} x_{i}-\log \left(\alpha_{1}\right) \sum_{i=1}^{n_{1}} e^{-\lambda_{1} x_{i}}-\log \left(\alpha_{2}\right) \sum_{i=1}^{n_{2}} e^{-\lambda_{2} x_{i}} \\
& +\sum_{i=1}^{n_{1}} \log \left(1-\alpha_{1}^{-e^{-\lambda_{1} x_{i}}}\right)+\sum_{i=1}^{n_{2}} \log \left(1-\alpha_{2}^{-e^{-\lambda_{2} x_{i}}}\right) .
\end{aligned}
$$

From (24), the MLEs of $\alpha_{k}$ and $\lambda_{k}, k=1,2$ can be obtained by solving the following likelihood equations

$$
\frac{\partial \log L}{\partial \alpha_{k}}=\frac{n}{\alpha_{k}}-\frac{n}{\alpha_{k}-1}+\frac{n_{k}}{\alpha_{k} \log \alpha_{k}}-\frac{1}{\alpha_{k}} \sum_{i=1}^{n_{k}} e^{-\lambda_{k} x_{i}}+\sum_{i=1}^{n_{k}} \frac{e^{-\lambda_{k} x_{i}} \alpha_{k}^{-\left(1+e^{-\lambda_{k} x_{i}}\right)}}{1-\alpha_{k}^{-e^{-\lambda_{k} x_{i}}}}
$$

and

$$
\frac{\partial \log L}{\partial \lambda_{k}}=\frac{n_{k}}{\lambda_{k}}-\sum_{i=1}^{n} x_{i}+\log \left(\alpha_{k}\right) \sum_{i=1}^{n_{k}} x_{i} e^{-\lambda_{k} x_{i}}-\sum_{i=1}^{n_{k}} \frac{x_{i} e^{-\lambda_{k} x_{i}} \alpha_{k}^{-e^{-\lambda_{k} x_{i}}} \log \left(\alpha_{k}\right)}{1-\alpha_{k}^{-e^{-\lambda_{k} x_{i}}}},
$$

where $k=1,2$. As it seems, equations (25) and (26) have no closed form solution in $\alpha_{1}, \alpha_{2}, \lambda_{1}$ and $\lambda_{2}$, so a numerical technique is used to solve these equation.

\subsection{Analysis of competing risks data set}

In this subsection, we analyze one data set which was originally analyzed by Hoel (1972), and later by many authors, see for example Kundu et al. (2004), Cramer and Schmiedt (2011) and Ashour and Nassar (2014, 2017). The data was obtained from a laboratory experiment in which male mice received a radiation dose of 300 roentgens at 35 days of age. The death cause for each mouse was classified by autopsy to be thymic lymphoma, reticulum cell sarcoma, or other causes. Here, for the analysis purpose we consider reticulum cell sarcoma as cause 1 and the other causes of death as cause 2 .

From the original data we have $n=77$ observations, where $n_{1}=38$ deaths due to cause one and $n_{2}=39$ deaths due to cause two. The MLEs of the unknown parameters are obtained and presented in Table 5 with the corresponding SEs. We compare the fitting of the APE distribution to the Hoel (1972) data with the exponential, Weibull and exponentiated exponential distributions. The estimates of the parameters of these distributions are given in Table 5 . We use the Akaike information criterion (AIC), and Bayesian information criterion (BIC) to compare the competitive models. The values of AIC and BIC are also displayed in Table 5. From Table 5, we conclude that the APE distribution provides better fits than the exponential, Weibull and exponentiated exponential distributions. 
Table 5: The MLEs (SEs in parentheses), AIC and BIC for Hoel (1972) data .

\begin{tabular}{|c|c|c|c|c|c|c|}
\hline Model & & Estin & nates & & AIC & $\mathrm{BIC}$ \\
\hline $\operatorname{APE}\left(\alpha_{1}, \alpha_{2}, \lambda_{1}, \lambda_{2}\right)$ & $\begin{array}{c}55975.26 \\
(47776.64)\end{array}$ & $\begin{array}{l}57.8499 \\
(58.725)\end{array}$ & $\begin{array}{c}0.0041 \\
(0.0003)\end{array}$ & $\begin{array}{c}0.0028 \\
(0.00044)\end{array}$ & .054 & 1143.429 \\
\hline $\mathrm{W}\left(\beta_{1}, \beta_{2}, \lambda_{1}, \lambda_{2}\right)$ & $\begin{array}{c}1.6330 \\
(0.4241)\end{array}$ & $\begin{array}{c}2.0083 \\
(0.1573)\end{array}$ & $\begin{array}{l}0.000046 \\
(0.00013)\end{array}$ & $\begin{array}{c}0.0000057 \\
(0.00001)\end{array}$ & 115 & 116 \\
\hline $\operatorname{EE}\left(\alpha_{1}, \alpha_{2}, \lambda_{1}, \lambda_{2}\right)$ & $\begin{array}{c}1.4218 \\
(0.3653)\end{array}$ & $\begin{array}{c}2.7256 \\
(0.6651)\end{array}$ & $\begin{array}{c}0.0031 \\
(0.0005)\end{array}$ & $\begin{array}{c}0.0050 \\
(0.00065)\end{array}$ & 1176.372 & 1185.748 \\
\hline $\mathrm{E}\left(\lambda_{1}, \lambda_{2}\right)$ & $\begin{array}{c}0.0026 \\
(0.00030)\end{array}$ & $\begin{array}{c}0.0032 \\
(0.00039)\end{array}$ & & & 1188.423 & 1193.111 \\
\hline
\end{tabular}

\section{Conclusion}

We discuss the estimation of the parameters of the alpha power exponential (APE) distribution using nine methods, namely, maximum likelihood estimation, moments estimation, L-moments estimation, ordinary least squares estimation, weighted least squares estimation, percentile estimation, maximum product of spacing estimation, Cramér-von Mises estimation and Anderson-Darling estimation. An extensive simulation study is performed to compare the performance of these methods. Overall, the simulation results reveal that the MPS method perform better than other methods in terms of MREs and RMSEs. Also, the results of moments and L-moments are unsatisfactory, therefore their results are excluded from the simulation study. We have also compared estimators by two real data applications. Using two real data applications, we show that the APE provides better fits than other revial models. Furthermore, real data set is used to show the flexibility of the APE distribution in modelling competing risks data.

\section{References}

1. Afify, A. Z. and Mohamed, O. A. (2020). A new three-parameter exponential distribution with variable shapes for the hazard rate: estimation and applications. Mathematics, 8, 1-17.

2. Afify, A. Z., Nassar, M., Cordeiro, G. M. and Kumar, D. (2020). The Weibull Marshall-Olkin Lindley distribution: properties and estimation. Journal of Taibah University for Science, 14, 192-204.

3. Afify, A. Z., Suzuki, A. K., Zhang, C. and Nassar, M. (2019). On three-parameter exponential distribution: properties, Bayesian and non-Bayesian estimation based on complete and censored samples. Commun. Stat.Simul. Comput. Doi:10.1080/03610918.2019.1636995.

4. Ashour, S. K. and Nassar, M. (2014). Analysis of exponential distribution under adaptive type-I progressive hybrid censored competing risks data. Pak. J. Stat. Oper. Res., 10, 2014.

5. Ashour, S. K and Nassar, M. (2017). Inference for Weibull distribution under adaptive type-I progressive hybrid censored competing risks data. Commun. Stat. Theory Methods, 46, 4756-4773.

6. Cheng, R. C. H., and Amin, N. A. K. (1979). Maximum product of spacings estimation with applications to the lognormal distribution. Technical report, Department of Mathematics, University of Wales.

7. Cheng, R. C. H., and Amin, N. A. K. (1983). Estimating parameters in continuous univariate distributions with a shifted origin. J. R. Statist. Soc. B, 45, 394-403.

8. Cordeiro, G. M., Afify, A. Z., Yousof, H. M., Cakmakyapan, S. and Ozel, G. (2018). The Lindley Weibull distribution: properties and applications. Anais da Academia Brasileira de CiÍncias, 90, 2579-2598.

9. Cramer, E. and Schmiedt, A. B. (2011). Progressively type-II censored competing risks data from Lomax distribution. Computational Statistics and Data Analysis, 55, 1285-1303.

10. D’Agostino, R. B. (1986). Goodness-of-fit-techniques. Statistics: A Series of Textbooks and Monographs. Taylor \& Francis.

11. Dey, A., Alzaatreh, A., Zhang, C. and Kumar, D. (2017). A new extension of generalized exponential distribution with application to ozone data. Ozone: Science \& Engineering, 39, 273-285.

12. Dey, S., Ghosh, I. and Kumar, D. (2019). Alpha-power transformed Lindley distribution: properties and associated inference with application to earthquake data. Annals of Data Science. Doi.org/10.1007/s40745018-0163-2.

13. Dey, S., Nassar, M. and Kumar, D. (2019). Alpha power transformed inverse Lindley distribution: a distribution with an upside-down bathtub-shaped hazard function. Journal of Computational and Applied Mathematics, 348, 130-145. 
14. Gupta, R. D. and Kundu, D. (2001). Generalized exponential distribution: different method of estimations. Journal of Statistical Computation and Simulation, 69, 315-337.

15. Hoel, D. G. (1972). A representation of mortality data by competing risks. Biometrics, 28, 475-488.

16. Hosking, J. R. M. (1990). L-Moments: analysis and estimation of distributions using linear combinations of order statistics. Journal of the Royal Statistical Society. Series B, 52, 105-124.

17. Jones, M. C. (2004). Families of distributions arising from distributions of order statistics. Test, 13, $1-43$.

18. Khan, M. S., King, R. and Hudson, I. (2017). Transmuted generalized exponential distribution: a generalization of the exponential distribution with applications to survival data. Commun. Stat. Simul.Comput., 46, 4377-4398.

19. Kundu, D., Kannan, N. and Balakrishnan, N. (2004). Analysis of progressivelycensored competing risks data. Handbook of Statistics, vol. 23, eds.,Balakrishnan, N. and Rao, C.R., Elsevier, New York.

20. Mahdavi, A. and D. Kundu. (2017). A new method for generating distributions with an application to exponential distribution. Commun. Stat. Theory Methods, 46, 6543-6557.

21. Mansoor, M., Tahir, M. H., Cordeiro, G. M., Provost, S. B. and Alzaatreh, A. (2018). The Marshall-Olkin logistic-exponential distribution. Commun. Stat. Theory Methods. Doi: 10.1080/03610926.2017.1414254.

22. Marshall, A. W. and Olkin, I. (1997). A new method for adding a parameter to a family of distributions with application to the exponential and Weibull families. Biometrika, 84, 641-652.

23. Mead, M. E., Cordeiro, G. M., Afify, A. Z. and Al Mofleh, H. (2019). The alpha power transformation family: properties and applications. Pak. J. Stat. Oper. Res., 15, 525-545.

24. Murthy, D. P., Xie, M. and Jiang, R. (2004). Weibull models. John Wiley \& Sons.

25. Nadarajah, S. and Kotz, S. (2006). The beta exponential distribution. Reliability engineering \& system safety, 91, 689-697.

26. Nadarajah, S. and Okorie, I. E. (2018). On the moments of the alpha power transformed generalized exponential distribution. Ozone: Science \& Engineering, 40, 330-335.

27. Nassar, M., Afify, A. Z., Dey, S. and Kumar, D. (2018a). A new extension of weibull distribution: properties and different methods of estimation. Journal of Computational and Applied Mathematics, 336, 439-457.

28. Nassar, M., Alzaatreh, A., Abo-Kasem, O., Mead, M. and Mansoor, M. (2018b). A new family of generalized distributions based on alpha power transformation with application to cancer data. Annals of Data Science, 5, 421-436.

29. Nassar, M. Alzaatreh, A., Mead, M. and Abo-Kasem, O. (2017). Alpha power Weibull distribution: properties and applications. Commun. Stat. Theory Methods. 46, 10236-10252.

30. Nassar, M., Dey, S. and Kumar, D. (2018c). A new generalization of the exponentiated Pareto distribution with an application. Amer. J. Math. Manag. Sci., 37, 217-242.

31. Nassar, M., Kumar, D., Dey, S., Cordeiro, G. M. and Afify, A. Z. (2019). The Marshall-Olkin alpha power family of distributions with applications. Journal of Computational and Applied Mathematics, 351, 41-53.

32. Sen, S., Afify, A. Z., Al-Mofleh, H. and Ahsanullah, M. (2019). The quasi xgamma-geometric distribution with application in medicine. Filomat, 33, 5291-5330.

33. Shakhatreh, M. K., Lemonte, A. J. and Cordeiro, G. M. (2019). On the generalized extended exponential-Weibull distribution: properties and different methods of estimation. International Journal of Computer Mathematics. DOI:10.1080/00207160.2019.1605062.

34. Weibull, W. (1951). A statistical distribution function of wide applicability. ASME Trans. J. Appl. Mech., 18, 293-297. 